Pleione 12(1): 87 - 93. 2018.

ISSN: 0973-9467

(c) East Himalayan Society for Spermatophyte Taxonomy

doi:10.26679/Pleione.12.1.2018.087-093

\title{
Sauromatum nangkarense (Araceae : Areae) - a new species from Arunachal Himalaya, India
}

\author{
Atek Nangkar and Hui Tag ${ }^{1}$ \\ Plant Systematics and Ethnobotanical Research Laboratory, Department of Botany, Rajiv Gandhi \\ University, Rono Hills, Doimukh-791112, Arunachal Pradesh, India. \\ ${ }^{1}$ Corresponding author, e-mail: huitag2008rgu@gmail.com
}

[Received 22.05.2018; Revised 19.06.2018; Accepted 23.06.2018; Published 30.06.2018]

\begin{abstract}
Sauromatum nangkarense A. Nangkar \& H. Tag, a new species of Sauromatum Schott, (Araceae) from Kimin, Papum Pare District of Arunachal Pradesh, India, is described and illustrated with photographs of living specimens. The new species resembles Sauromatum brevipes and $S$. hirusutum with their staminodes short and curved downward and the spathe-tube is convulated. The new species differs from these in having subcylindric tubers, pinnate leaves, with male zone blonde, cream, sterile flowers clavate, pink, stipitate and curved downward covering female zone partially. Detailed description and a range of photographs have been provided.
\end{abstract}

Key words: Sauromatum nangkarense, New species, Araceae, Kimin, Arunachal Pradesh, India

\section{INTRODUCTION}

Sauromatum Schott, (Araceae; Areae) is a genus with nine accepted species (www.theplantlist.org). Genus Sauromatum and Typhonium Schott are overlapping genera as many species are sometimes placed in both of these by different workers. Hetterscheid and Boyce in 2000 clearly circumscribed these two distinct genera. The genus Sauromatum is distributed in South, Southeast and East Asia and extends throughout the pacific area to Australasia. In India the genus is represented by four species, viz. Sauromatum brevipes (Hook.f.) N.E. Brown, S. venosum (Dryand. ex Ait.) Kunth, $S$. diversifolium (Wall. ex Schott) Cusimano \& Hett. (Cusimano et al. 2010), and one recently published species $S$. meghalayense D.K. Roy, A.D. Talukdar, B.K. Sinha \& M. Dutta Choudhury (Roy et al. 2014).

During intensive floristic survey in Arunachal Pradesh, India, the authors collected one interesting specimens from the Kimin area of Papum Pare district. The plant was also observed growing in tropical belts of Siang and Kameng belts of the state, mostly occurring in tropical forest along the low altitude foothills, up to $400 \mathrm{~m}$ amsl. The significant morphological characters of the mature plant i.e. pedatisect leaf blade, inflorescence appearing along with developing leaf, short peduncle, spathe tube with convoluted margins, sterile-zone with structure of staminodes, stipitate appendix cone or knife shaped, and tube persistent. These morphological characters showed that collected plant is a species Sauromatum Schott. But, the morphological characters of its tuber, leaf blade, petiole, spathe tube, spadix, structure of staminodes, male and female zone, stipitate appendix, and bifid pointed stigma made it is distinct from the so far known species of the genus (Hooker 1894; Cusimano et al. 2010). 
So, the plant is now recognized as a new species of Sauromatum Schott for which description, illustration, colour photographs, distributional details, and type specimen are provided below.

This taxon is quite close to $S$. venosum but differs in having short inflorescence appearing alongside first developing leaf, spreaded limb with trunicate apex, two types of staminode, appendix conic and stipitate. The new species can also be distinguished from $S$. brevipes in having stipitate and conic appendix, dark purple-brown peduncle; from $S$. hirsutum (S.Y. Hu) Cusimano \& Hett. and S. brevipilosum (Hett. \& M. Sizemore) Cusimano \& Hett. in having glabrous petiole and leaf blade; purple brown spot on petiole, stipitate appendix, bipid with pointed stigma; from Sauromatum meghalayense D.K.Roy, A.D.Talukdar, B.K.Sinha \& M. Dutta Choudhury. In having, erect peduncle, dark purple brown spot on petiole, bipid pointed stigma, subcylindric tuber, barries green, A comparison of the morphological characters of $S$. nangkarense with $S$. brevipes, $S$. hirsutum, and $S$. meghalayense is given in Table 1.

\section{TAXONOMY}

\section{Sauromatum nangkarense A.Nangkar \& H.Tag, sp. nov. [Figures 1 - 4]}

Herbs, terrestrial tuberous herbs, monoecious. tubers globose to subcylindric, annulate, $4 \mathrm{~cm}$ long, $3 \mathrm{~cm}$ wide. Roots: adventitious, terete, white, arise from near the base of petiole/ pseudostem. Cataphylls 2, dark purple to pinkish, lanceolate, acute to acuminate, $9 \mathrm{~cm}$ long and $5 \mathrm{~cm}$ wide. Leaves: solitary, occasionally 3 ; petiole green, dark purple spotted, $20-40$ $\mathrm{cm}$ long, $1-2 \mathrm{~cm}$ wide; Leaf blade: pedatifid, segments $5-7$, green, simple, oblong or oblanceolate or elliptic, entire, acute to acuminate, central segments large, $14-20 \times 8-10$ $\mathrm{cm}$, lateral two leaflets smallest, $7-12 \times 3-5 \mathrm{~cm}$, gradually smaller toward the distal end of rachis, first two lateral veinlets acts as end vein collector, $2-3$ line of end vein collectors on leaf margins united at the apex. Inflorescence: solitary, arise along with leaves. Peduncle: green, dark purple brown spotted, $2.5-5 \times 0.5 \mathrm{~cm}$. Spathe: convolute at base. Tube: subglobose, glabrous, slightly constricted, dark purplish violet, margins overlap each other to make tube, ovoid or ellipsoid, $3.7-5 \mathrm{~cm}$ long and $4 \mathrm{~cm}$ wide, pinkish inside, female flowers closed inside the tube, tube persistent; limb $9 \mathrm{~cm}$ long, $16 \mathrm{~cm}$ wide, spreading like elephant ear, both sides light green with purple, later on white, fall off in later stage. Spadix: sessile; female part $3.2 \mathrm{~cm}$ long, $2.5 \mathrm{~cm}$ wide, conic, ovaries green, generally 1-loculed, rarely 2loculed, stigma white, subsessile, apex pointed. $1^{\text {st }}$ sterile zone: $0.6 \mathrm{~cm}$ long, white to pink, proximal part covered with staminodes, distal part groove and naked. Staminodes: thick, purple, $2.2 \mathrm{~cm}$ long, $0.3 \mathrm{~cm}$ wide, curved downward to covered almost $80-90 \%$ of the female zone, clavate, and emarginate, apex v-shape, arise from centre of ridge. Male zone yellow, blonde, cylindrical, $2 \mathrm{~cm}$ long, $0.5 \mathrm{~cm}$ wide. Appendix ( ${ }^{\text {nd }}$ sterile zone): stipitate, white-daisy, slightly conic or knife shaped, apex obtuse, $7 \mathrm{~cm}$ long, $0.6 \mathrm{~cm}$ wide at base. Stipe $0.7 \mathrm{~cm}$ long, $0.3 \mathrm{~cm}$ wide, daisy, pink. Berries crowded, subglobose to banana shaped, young green to mature dark purple.

Flowering \& Fruiting: March - September

Phenology: seeds germinate in the month of April, generally $80-90 \%$ seeds germinate; in the first year eophil with one leaf with sagittate blade to trifoliate with shallow lobes appear; takes $2-3$ years to mature. Generally flowers in the $4^{\text {th }}$ year march - April; leaves wither during August - September when fruits also found to ripe.

Type: INDIA, Arunachal: Kemin, Papum Pare District. Kemin, 28.04.2015, Atek Nangkar \& Hui Tag 055 (Holotype: ASSAM; Paratype: HAU). 


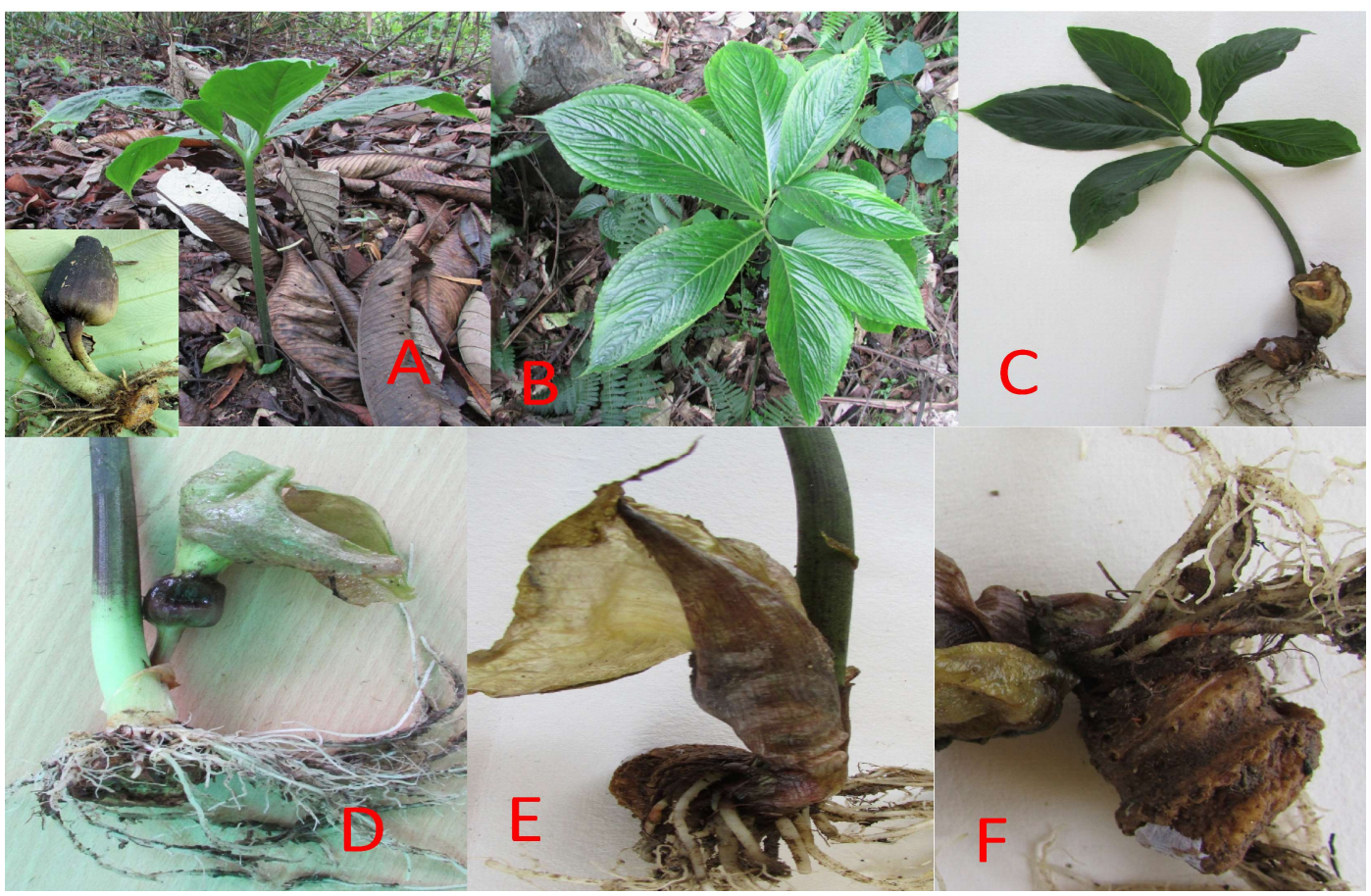

Figure 1. Sauromatum nangkarense A.Nangkar \& H.Tag, sp. nov. A. whole plant with lower part of spathe in fruiting stage; B. pedately divited leaf; C. plant with inflorescence ; D-E. Inflorescence in close-up view; F. tuber with roots - lower part shows the last year's degrading part and upper part is the current year's.

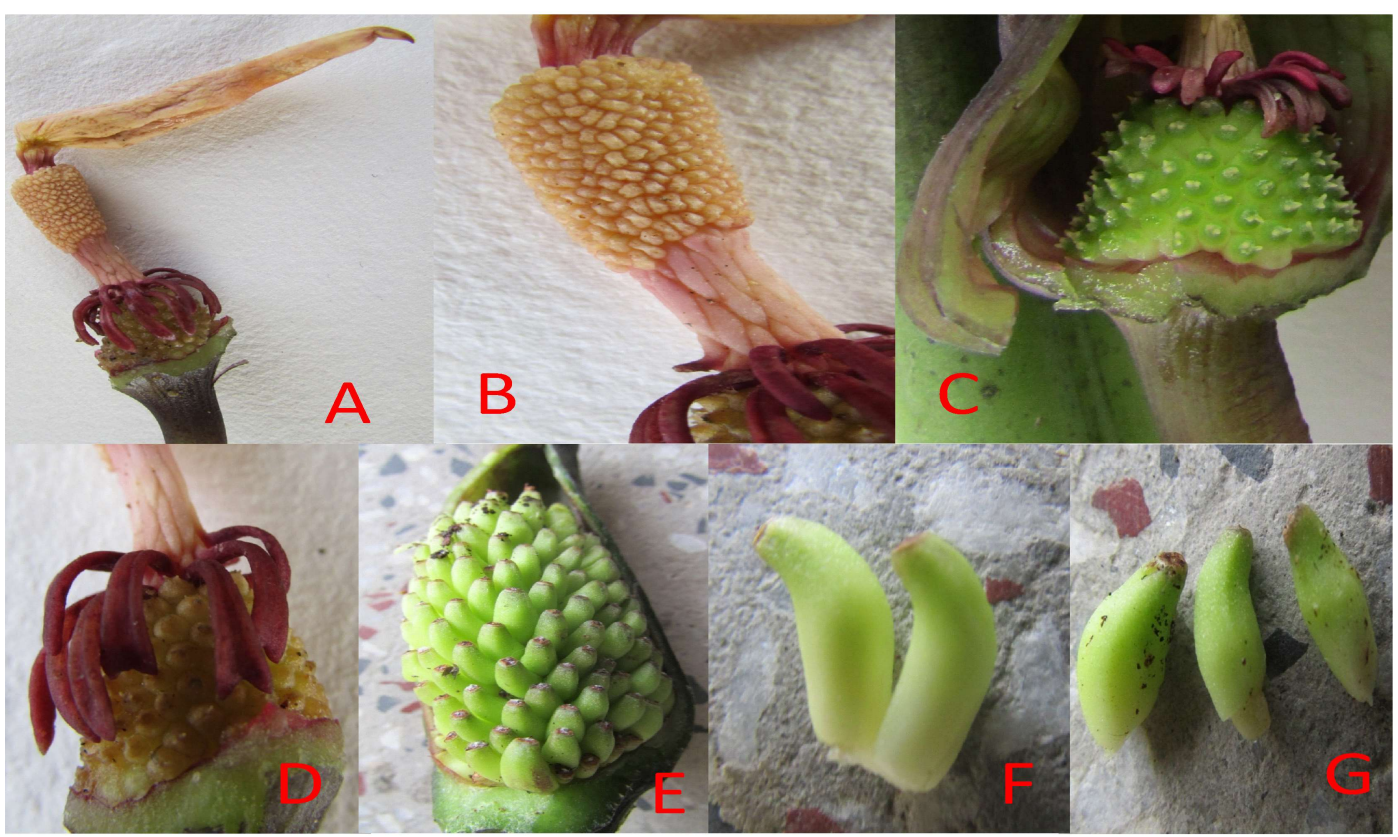

Figure 2. Sauromatum nangkarense A.Nangkar \& H.Tag, sp. nov. A. spadix; B. male flower zone; C. female flower zone; D. staminodes; E. infructescense; F \& G. isolated fruits 


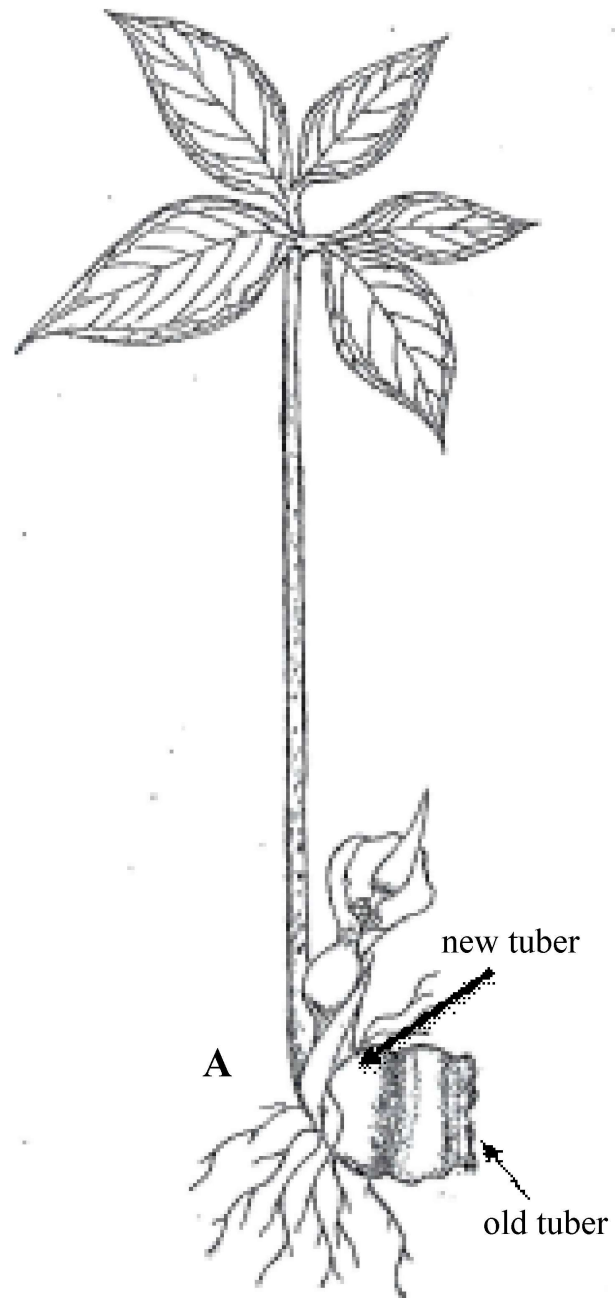

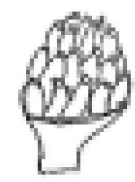

C

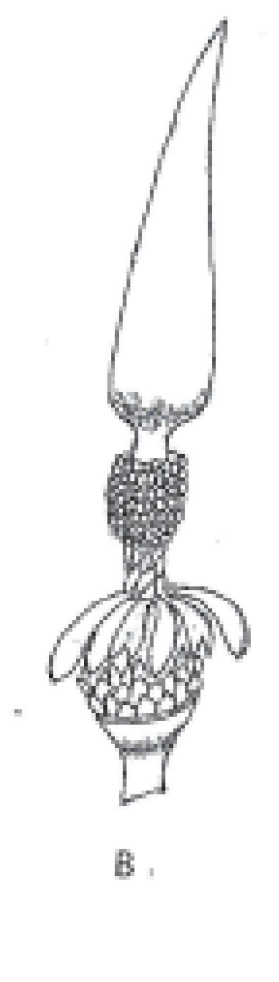

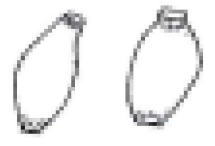

D
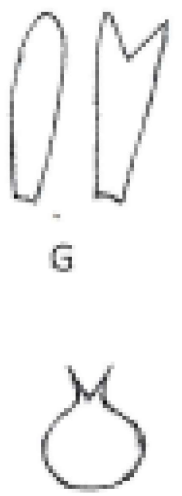

E.

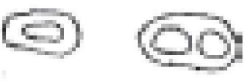

F

Figure 3. Sauromatum nangkarense A.Nangkar \& H.Tag sp. nov. A. whole plant with leaves and inflorescence; B. Spadix; C. Fruits; D. Seed; E. overy with stigma; F. T.S of ovule show single and double loculed; G. Staminodes

Distribution: Kimin: Papum Pare District and also observed from five district of Arunachal Pradesh, India; endemic.

Habitat: Grows in tropical evergreen broadleaved forest floor, secondary degraded forest, open wet area and grassland at an altitudinal range of $150-400 \mathrm{~m}$.

Conservation status: seen to grow in tropical broadleaved evergreen forest, secondary degraded forest, open wet area and grassland in five districts of Arunachal Pradesh. Following IUCN (2001), criteria this taxon should therefore be treated as Data Deficient.

Etymology: The specific epithet nangkarense indicates the dynasty of the first Author and after the name of his father late Kuyang Nangkar, belongs to Adi tribe of Arunachal Pradesh, India.

Note: An artificial key to identify the Indian species of Sauromatum Schott has been prepared. The differences of $S$. nangkarense from its close alleys $S$. brevipes, $S$. hirsutum, and $S$. meghalayanse is presented in Table 1. 

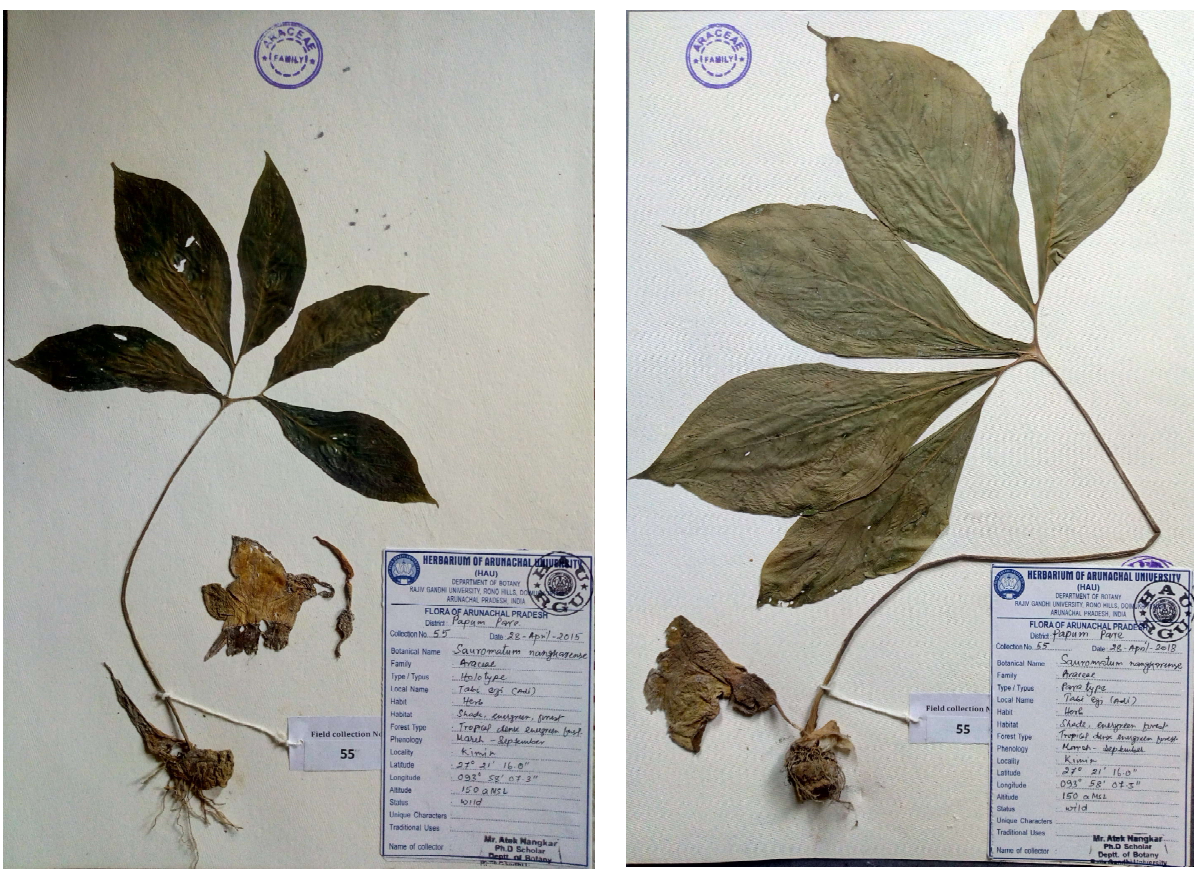

Figure 4. Herbarium specimens Sauromatum nangkaarense. A. Whole plant with separate spathe \& spadix. B. Whole plant with inflorescence.

Table 1. Comparison of diagnostic morphological characters of Sauromatum nangkarense and its related species.

\begin{tabular}{|c|c|c|c|c|}
\hline Characters & $\begin{array}{l}\text { Sauromatum } \\
\text { brevipes }\end{array}$ & $\begin{array}{l}\text { Sauromatum } \\
\text { hirsutum }\end{array}$ & $\begin{array}{l}\text { Sauromatum } \\
\text { meghalayense }\end{array}$ & $\begin{array}{l}\text { Sauromatum } \\
\text { nangkarense }\end{array}$ \\
\hline tuber & $\begin{array}{l}\text { Globose, } 1-1.5 \mathrm{~cm} \\
\text { in diam. }\end{array}$ & $\begin{array}{l}\text { Subglobose, } 1.5-2.5 \\
\mathrm{~cm} \text { in diam. }\end{array}$ & $\begin{array}{l}\text { Globose, to } 3.5 \mathrm{~cm} \\
\text { high, } 4 \mathrm{~cm} \text { in diam. }\end{array}$ & $\begin{array}{l}\text { Subglobose- } \\
\text { subcylindric, } \\
\text { annulate, } 4 \mathrm{~cm} \\
\text { long, } 3 \mathrm{~cm} \text { diam. }\end{array}$ \\
\hline petiole & $\begin{array}{l}\text { Without hair, } \\
\text { pinkish or dull } \\
\text { purplish red, } 15- \\
30 \mathrm{~cm} \times 2 \mathrm{~mm} .\end{array}$ & $\begin{array}{l}\text { Densely hirsute, } 15- \\
20 \mathrm{~cm} \text { long. }\end{array}$ & $\begin{array}{l}\text { Without hair, } \\
\text { greenish, to } 45 \mathrm{~cm} \\
\text { long, } 1.7 \mathrm{~cm} \text { in } \\
\text { diam }\end{array}$ & $\begin{array}{l}\text { Without hair, } \\
\text { touch rough, } \\
\text { Petiole pale } \\
\text { green with dark } \\
\text { purple spot or } \\
\text { dark purple } \\
\text { brown, } 20-40 \mathrm{~cm} \\
\text { long, } 1-2 \mathrm{~cm} \\
\text { diam. }\end{array}$ \\
\hline Leaf blade & $\begin{array}{l}\text { Glabrous, 5-9- } \\
\text { pedatifid; central } \\
\text { lobe linear- } \\
\text { lanceolate or } \\
\text { narrowly elliptic, }\end{array}$ & $\begin{array}{l}\text { Densely hirsute, } 5- \\
\text { 7-pedatified; central } \\
\text { lobe elliptic, 7-31 x } \\
2.5-10 \mathrm{~cm}\end{array}$ & $\begin{array}{l}\text { Glabrous,7- } \\
\text { pedatified; central } \\
\text { lobe oblanceolate, } \\
33 \times 8 \mathrm{~cm} .\end{array}$ & $\begin{array}{l}\text { glabrous, 5-7 } \\
\text { pedatifid. } \\
\text { Centeral lobe } \\
\text { elliptic, oblong } \\
\text { to lannenlate } 14\end{array}$ \\
\hline
\end{tabular}




\begin{tabular}{|c|c|c|c|c|}
\hline Characters & $\begin{array}{l}\text { Sauromatum } \\
\text { brevipes }\end{array}$ & $\begin{array}{l}\text { Sauromatum } \\
\text { hirsutum }\end{array}$ & $\begin{array}{l}\text { Sauromatum } \\
\text { meghalayense }\end{array}$ & $\begin{array}{l}\text { Sauromatum } \\
\text { nangkarense }\end{array}$ \\
\hline & $\begin{array}{l}\text { narrowly elliptic, } \\
6.5-25 \times 1-5 \mathrm{~cm} .\end{array}$ & & & $\begin{array}{l}\text { to lanceolate, } 14 \\
-20 \mathrm{~cm} \text { long, } 8- \\
10 \mathrm{~cm} \text { wide. }\end{array}$ \\
\hline Inflorescence & $\begin{array}{l}\text { Inflorescence(s) } \\
\text { appearing } \\
\text { alongside } \\
\text { developing leaves. }\end{array}$ & $\begin{array}{l}\text { Inflorescence } \\
\text { appearing alongside } \\
\text { first developing leaf. }\end{array}$ & $\begin{array}{ll}\text { Inflorescence } & \\
\text { appearing } & \\
\text { alongside } & \text { first } \\
\text { developing } & \text { leaf, } \\
\text { curving } & \end{array}$ & $\begin{array}{ll}\text { Inflorescence } \\
\text { appearing } \\
\text { alongside first } \\
\text { developing } & \text { leaf, } \\
\text { erect } & \text { (not } \\
\text { curved) } & \end{array}$ \\
\hline Peduncle & $\begin{array}{l}\text { Pale green, } 2-2.5 \\
\mathrm{~cm} \text { long. }\end{array}$ & $1-1.5 \mathrm{~cm}$ long & $\begin{array}{l}\text { Purplish-brown, } \\
\text { ca. } 5 \mathrm{~cm} \text { long, } 0.6 \\
\mathrm{~mm} \text { in diam. }\end{array}$ & $\begin{array}{l}\text { green, dark } \\
\text { purple brown, } \\
2.5-5 \mathrm{~cm} \text { long } \\
\text { and } 0.5 \mathrm{~cm} \text { diam. }\end{array}$ \\
\hline Spathe tube & $\begin{array}{l}\text { Yellowish-brown } \\
\text { with purple or } \\
\text { faint pinkish spots } \\
\text { outside, creamy or } \\
\text { dark pink inside; } \\
\text { margin closed. }\end{array}$ & $\begin{array}{l}\text { Hirsute outside; } \\
\text { convolute but } 1 \mathrm{~cm} \\
\text { with fused margin, } \\
\text { purplish red inside. }\end{array}$ & $\begin{array}{l}\text { Smooth both side; } \\
\text { convolute with } \\
\text { open margins, } \\
\text { purplish but } \\
\text { creamy to white in } \\
\text { margins and } \\
\text { toward the limp } \\
\text { inside. }\end{array}$ & $\begin{array}{l}\text { dark purplish } \\
\text { violet inside } \\
\text { pinkish, ovoid } \\
\text { or ellipsoid, } \\
3.7-5 \mathrm{~cm} \text { long, } \\
8 \mathrm{~cm} \text { wide }\end{array}$ \\
\hline
\end{tabular}

\section{Keys to the Indian species of Sauromatum}

1a. Leaf blade diversified (often in one plant), entire to pedatisect ......S. diversifolium

1b. Leaf blade always pedatisect ................................ 2

2a. Inflorescence appearing before leaves ........................ S. venosum

2b. Inflorescence appearing along with first developing leaf ' '........... 3

3a. Peduncle pale green, $2-2.5 \mathrm{~cm}$; spathe tube greenish to white inside ... S. brevipes

3b. Peduncle purplish-brown or with dark purple spots ................ 4

4a. Peduncle purplish brown, ca. $5 \mathrm{~cm}$; spathe tube purplish but creamy to white in margins and toward the limp inside; appendix not stipited ......... S. meghalayense

4c. peduncle dark purple, $2.5-5 \mathrm{~cm}$; spathe dark purplish violet, pinkish inside; appendix stipitate .S. nangkarense

\section{Acknowledgements}

The authors are thankful to UGC New Delhi for funding support through CPEB-II project. Authors deeply acknowledges the valuable technical help rendered by Prof. A. P Das, DBT Visiting Research Professor, Department of Botany, Rajiv Gandhi University during development of this manuscript. 


\section{LITERATURE CITED}

Cusimano, N.; Barrett, M.D.; Hetterscheid, W.L.A. \& Renner, S. 2010. A phylogeny of the Areae (Araceae) implies that Typhonium, Sauromatum and the Australian species of Typhonium are distinct clades. Taxon 59(2): $439-447$

Hettercheid, W.L.A. \& Boyce, P.C. 2000. A reclassification of Sauromatum Schott and new species of Typhonium Schott (Araceae). Aroideana 23: 48 - 55.

IUCN 2001. IUCN Red List Categories and Criteria: Version 3.1. IUCN Species Survival Commission. IUCN, Gland, Switzerland and Cambridge, U.K.

Hooker, J.D. 1894. The Flora of British India. L. Reeve and Co., London. Vol. 6. pp. 488556.

Roy, D.K.; Talukdar, A.D.; Sinha, B.K. \& Dutta Choudhury, M. 2014. Sauromatum meghalayense (Araceae; Tribe: Areae), a new species from Meghalaya, India. NeBIO 5(3): $1-3$.

www.theplantlist.org 